\title{
Scattering of free electrons by free electrons
}

\author{
J. F. Williams, ${ }^{1, *}$ S. Samarin, ${ }^{1}$ O. Targhagh, ${ }^{1}$ A. Hilton, ${ }^{1}$ K. Sudarshan,,${ }^{1,}$ P. Guagliardo, ${ }^{1}$ L. Pravica, ${ }^{1}$ and O. Artamonov ${ }^{2}$ \\ ${ }^{1}$ ARC Centre of Excellence for Antimatter and Matter Studies, Centre for Atomic, Molecular and Surface Physics, School of Physics, \\ The University of Western Australia, Crawley, Perth WA6009, Australia \\ ${ }^{2}$ Department of Physics, St. Petersburg University, St. Petersburg, Russia
}

(Received 1 September 2013; published 25 June 2014)

\begin{abstract}
The scattering of single free electrons by free electrons has been measured directly. The methods of measurement included changing the incident relative velocities of the electrons by changing either the angle between the beams or the beam energies and then identifying uniquely the outgoing electron pairs with energy and coincidence time detection. The measured energy and angular differential scattering cross sections over the relative interaction velocity range from $0.3 \times 10^{7}$ to $3.0 \times 10^{7} \mathrm{~m} / \mathrm{s}$ for forward and backward scattering angles which were calibrated absolutely to within $\pm 2 \%$ and with a statistical uncertainty within $\pm 2 \%$ at an angle of $30^{\circ}$ between the beams and an observational angle of $60^{\circ}$. Subsequent relative data showed a dependence on (relative velocity $)^{-4}$ and $\cos ^{2}$ (beam intersection angle) scattering parameters as expected for an exact quantum mechanical description for pure Coulomb field Møller scattering. While the precision and reproducibility of all data are at the percent level, four of the 48 data points vary significantly from the expected quantum values and are not included in our overall experimental uncertainty.
\end{abstract}

DOI: 10.1103/PhysRevA.89.062717

PACS number(s): $34.80 .-\mathrm{i}$

\section{INTRODUCTION}

The interaction of electrons with other electrons appears in many different ways in all forms of matter and provides vastly different information about many fundamental aspects of science. Examples in physics range from parity violation in electron-electron Møller scattering at $\mathrm{GeV}$ energies [1] and plasma screening in dense high-temperature plasmas [2] to Auger electron characterization of materials [3], electron correlations and polarization phenomena of electron densities in excited atomic states [4], and electron transport in lowdimensional quantum materials at low energies [5]. Generally the high-energy electron scattering processes have been relatively well described by Møller scattering and quantum electrodynamics [1] and in the other environments by various quantum approximation methods, such as self-consistent Hartree-Fock theory [6] and density functional methods [7].

Almost everything we know about nuclei and elementary particles has been discovered in scattering experiments and the properties of condensed matter rely fundamentally on scattering methods. Even though it is well established that electrons can behave as point particles and have an infinite range of their Coulomb potential, there was need for more studies involving the direct measurement of their mutual scattering. Also when direct observations cannot be made for particle scattering, it is usual to apply a first Born approximation (FBA) as a reasonable initial guide to the interaction, particularly when velocities are high [8]. The present work describes the experimental procedures and determines calibrated differential cross sections with the measured parameters of electron velocities and scattering angles and confirms such, within an experimental uncertainty of $\pm 4 \%$, and shows the ability of precision measurements of free-free electron scattering

\footnotetext{
*Corresponding author: jim.williams@uwa.edu.au

${ }^{\dagger}$ On leave from Radiochemistry Division, Bhabha Atomic Research Centre, Mumbai, India 400085.
}

averaged over the electron spin to obtain data in agreement with the exact quantum description [8].

\section{EXPERIMENTAL CONSIDERATIONS}

\section{A. Design}

Our approach for an absolute calibrated single particle scattering measurement uses the method of intersecting charged particle beams $[9,10]$ which allows full differential measurements of the energy and angular cross sections. For collisions of equal mass and equal charge particles, here electrons, the scattering dynamics can be considered in velocity space. The angular transformations from the center-of-mass (c.m.) coordinates $\left(\theta_{0}, \phi_{0}\right)$ to the laboratory measuring coordinate $(\theta, \phi)$ system [11] are straightforward but different compared with those of unequal masses encountered in electron-atom scattering.

Figure 1 indicates the laboratory coordinate reference frame with velocity vectors $\mathbf{V}_{\mathbf{1}}$ and $\mathbf{V}_{\mathbf{2}}$ of the two incident electron beams in the scattering $(X, Z)$ plane and at equal angles $\psi$ with respect to the positive and negative vertical $Z$ axis, respectively. Then the relative velocity $V_{R}$ of the incident beams is given by

$$
V_{R}^{2}=V_{1}^{2}+V_{2}^{2}-2 V_{1} V_{2} \cos (\pi-2 \psi),
$$

and can be varied by changing the angle $(\pi-2 \psi)$ or the magnitudes of the velocities. For the scattered electron velocity vectors located by angles $(\theta, \phi)$, the $\phi$ angle is used in this work only to identify forward and backward scattering with $\phi=0^{\circ}$ and $180^{\circ}$, respectively.

The representation of this scattering process and the transformation from c.m. to laboratory coordinates is indicated best for the case of equal velocity electrons, as in Fig. 2. In the c.m. frame the two electrons always move in opposite directions with velocities $V_{R} \cos \phi$. In the laboratory frame, as the angle $\phi$ increases, the relative velocity decreases while the center of mass of the incident electrons before scattering 


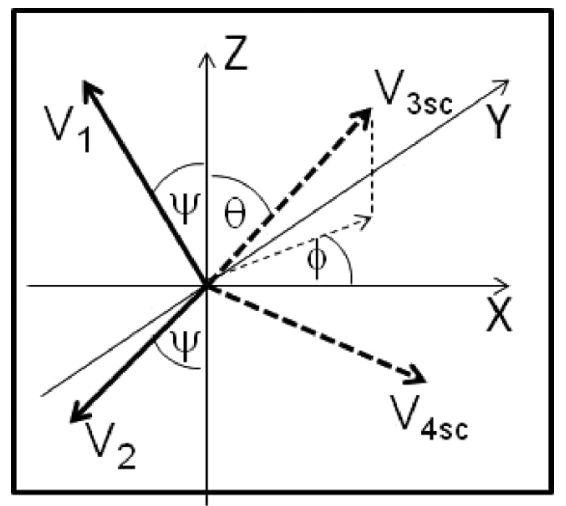

FIG. 1. The laboratory orthogonal rectilinear $(X, Y, Z)$ coordinate system. The initial velocity vectors $V_{1}$ and $V_{2}$ of the electrons make angles $\psi_{1}$ and $\psi_{2}$, respectively, with respect to the $Z$ axis. The scattered velocity vectors $V_{3 s c}$ and $V_{4 s c}$ (dashed lines) are located at angle $(\theta, \phi)$ in a normal right-handed orthogonal coordinate frame.

moves to the left with velocity $V_{R} \sin \phi$ and after scattering moves to the right with velocity $V_{R} \sin \phi$. Also, as the angle $\psi$ increases, the constant detector solid angle will observe a larger angular range of c.m. scattering because the ratio of c.m. solid angle to the viewed laboratory solid angle increases significantly.

The transformation between coordinate systems of both the scattering angle $\theta$ and the solid angle is obtained readily by consideration of the classical velocity vector geometry in Fig. 2.

For backward directions $\left(\phi_{0}=180^{\circ}\right)$, the angular transformation is given by

$$
\tan \theta=\left(\tan \psi-\sin \theta_{0}\right) \sec \theta_{0},
$$

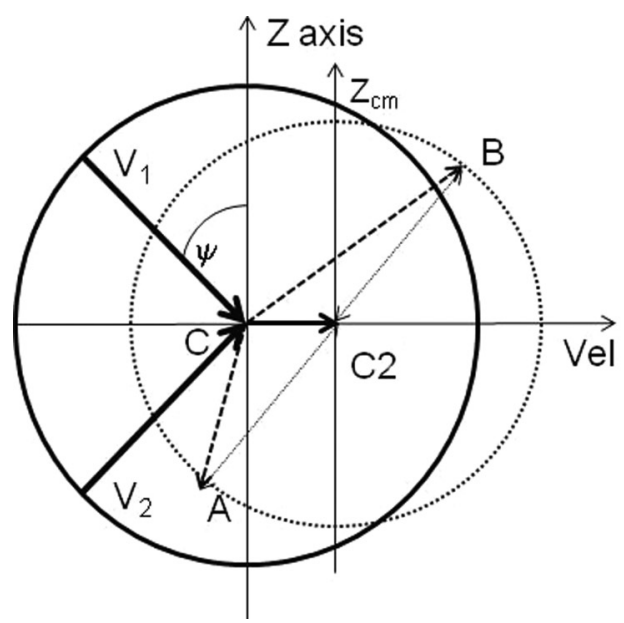

while for forward directions $\left(\phi_{0}=0^{\circ}\right)$ the terms are the same but the signs are always positive. For solid angles for backward directions,

$$
\Delta \Omega_{0} / \Delta \Omega=\left[\tan ^{2} \psi-2 \tan \psi \sin \theta_{0}+1\right]^{3 / 2}\left[1-\tan \psi \sin \theta_{0}\right]^{-1},
$$

and for forward angles the terms are again the same but the signs are all positive. The behavior of Eq. (3) is determined by whether the product $\left[\tan \psi \sin \theta_{0}\right]$ is greater than or equal to 1 , or less than 1 , that is effectively indicating that the center of mass has moved away from the laboratory (observed) scattering center sufficiently for the observed scattering to change from both forward and backward angles to only forward scattering angles. The derivation of Eq. (3) is helped by noting in Fig. 2 that in c.m. frame from the definition of solid angle $\Delta \Omega_{\mathrm{o}}=\Delta S[V \cos \psi]^{-2}$, and in the laboratory frame $\Delta \Omega_{\text {lab }}=\Delta S(C B)^{-2} \cos (C B A)$ where $\Delta S$ is an element of area on the surface, $C B$ is the scattered electron velocity, and the angle $C B A$ follows from geometry.

The differential cross section $d \sigma(\theta, \phi) / d \Omega$ is determined from the scattered electrons $/ \mathrm{s} I_{s}(\theta, \phi)$ scattered into solid angle $\Delta \Omega$ where

$$
d \sigma(\theta, \phi) / d \Omega=I_{s}(q, \phi) \Delta \Omega\left[(n A V)_{1}(n A V)_{2} F d \varsigma\right]^{-1}
$$

and $(n A V)_{1,2}$ are the effective number densities, crosssectional area, and velocity of the beams, $F$ is the fraction of $d \varsigma$, the intersection volume viewed by the detector. All these quantities are measurable; hence absolute values of the cross sections were determined.

\section{B. Instrumentation}

The basic apparatus may be described as a highresolution electron energy loss spectroscopic (HREELS)

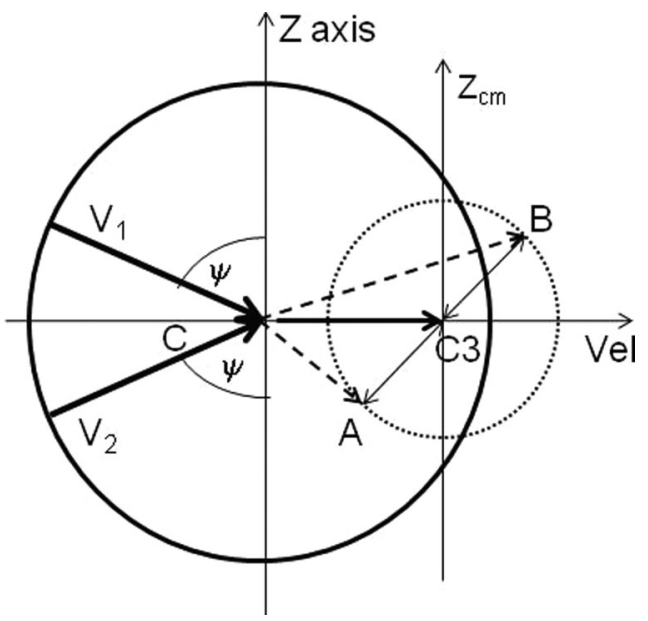

FIG. 2. The velocity vector representation of the scattering of two free electrons with velocities $V_{1}$ and $V_{2}$ at angles $\psi_{1}$ and $\psi_{2}$, respectively, to the $Z$ axis in the laboratory coordinate frame. For the coplanar case of $V_{1}=V_{2}$ and $\psi_{1}=\psi_{2}$ (for simplicity of the figure), the motion of the c.m. relative to the laboratory frame is indicated. The centers of the two dotted circles indicate the motion of the centers of mass (in laboratory frame) with velocities $V_{R} \sin \psi$ while their radii of $V_{R} \cos \psi$ (thin lines) indicate the magnitudes of the velocities after scattering in the c.m. coordinates, respectively. As the angle $\psi$ increases, the center of mass moves with velocity $V_{R} \sin \psi$ from $C 2$ to $C 3$. The velocity axis is scaled to the ratio of scattered to incident velocity. The dashed lines from $C$ indicate the observed (laboratory) scattering velocity vectors such that $C A$ and $C B$ change from forward and backward scattering to only forward scattering when $\psi$ is greater than $45^{\circ}$. Note that the vectors $B C$ and $B C 2$, and also $B C$ and $B C 3$, make angles $\theta$ and $\theta_{\mathrm{o}}$ with respect to the vertical $Z$ axes. Also the angles $(C B A)$ are indicative of the change of solid angle from c.m. to laboratory frame. 


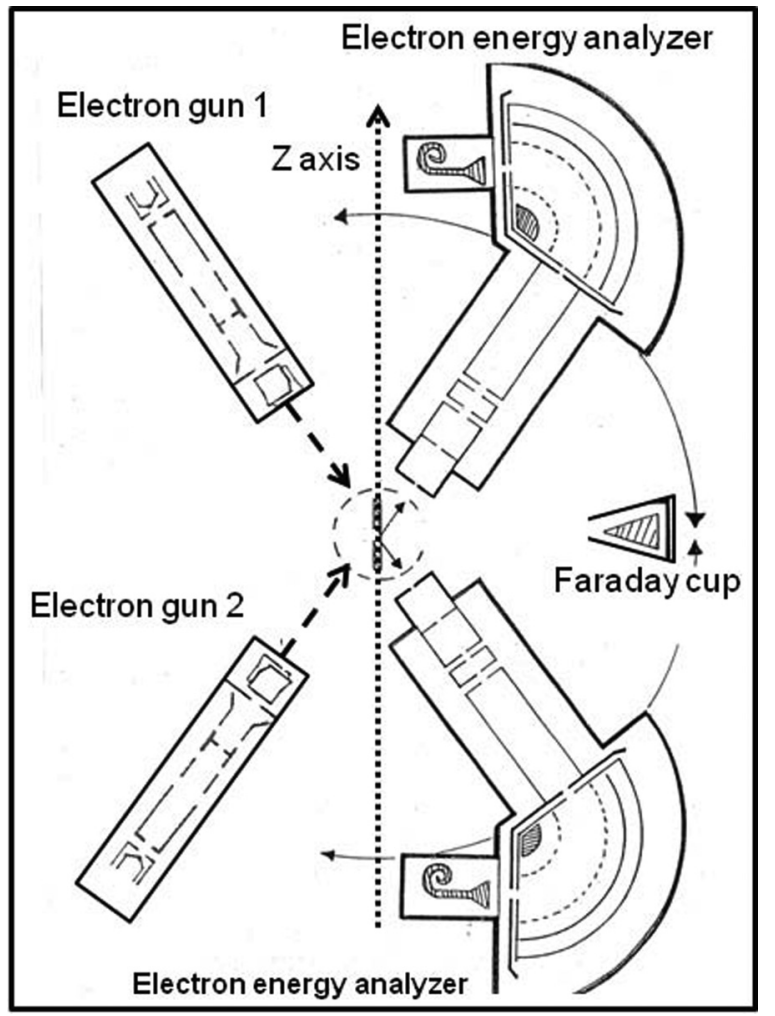

FIG. 3. A representation, not to scale, of the electron scattering planar geometry in the $Z X$ plane as indicated in Fig. 1. The two electron guns and two electron energy analyzers are identical and all can be moved independently radially and rotated around the laboratory scattering center defined by the intersection of the two electron beams. The radial motion allows the subtended entrance solid angles to the detectors to be adjusted and allows a larger range of rotational angles. Details of the electron analyzers and their characteristics are given in [15].

design instrument using two $127^{\circ}$ cylindrical electron energy analyzers as represented in Fig. 3. The electron scattering instrumentation had been designed and used for electron scattering studies [12] and was adapted to the characteristics of intersecting electron beams with up to keV laboratory energies and about $10^{-10}$ Torr UHV. Pertinent aspects of the apparatus, changed from earlier reports, are described briefly. The electron energy analyzers, electron beams, and their collectors (Faraday cups designed for extremely low reflection properties) were located so that each could be rotated and/or moved radially, from outside the UHV system, to access the parameters used in Eqs. (1)-(3). The central scattering region could be viewed by fluorescent screens, which could be moved in or out of the scattering plane. The beam profiles of Eq. (4) were measured by transverse scans of a sharp edge.

Standard five-element electrostatic lenses with variable electron optics, and collimating apertures, together with electrostatic beam-modulating deflection plates, established beam transport optics. The scattered electrons were selected by the acceptance solid angle of identical $127^{\circ}$ electron energy analyzers with well known characteristics [12] and with a beam energy resolution of about $1 / 30$ of their pass energies (determined by their mean radius and slit widths).
The energies of the scattered electrons were retarded usually to enable an analyzer pass energy of $20 \mathrm{eV}$ with a resolution of $0.7 \mathrm{eV}$.

The identification of the scattered electron pairs was made uniquely by electron energy analyzer selection of both scattered electrons and their coincidence detection [13-15]. For the largest cross sections, the channel electron multiplier detectors counted up to the order of $5 \times 10^{7}$ counts $/ \mathrm{s}$, provided the electronic noise was not greater than about $2 \mathrm{mV}$ and the reduced pulse height distribution and the counter dead times accompanying higher count rates were identifiable. Solid coaxial cables from the detectors were essential for noise reduction. The pulse counting Ortec electronics were standard modules. In summary, electron-electron time coincidence events were recorded with a signal-to-noise ratio that varied up to orders of magnitude and in accordance with well established procedures $[13,16]$.

The geometry and mechanical changes require observation of scattering from a beam intersection angle of near zero, i.e., overlapping beams, for about $30 \mathrm{~mm}$, which was tedious and used only for one series of measurements for the present study to indicate proof of principle. This experimental capability is explored in future work for electron-ion scattering [17] and is an extension of a similar use of electron beams of different energies transported through a single electrostatic field $[18,19]$.

The validity of the measurements rested upon the demonstration that the measured quantities were really the physical quantities in Eqs. (1)-(4) and the accuracies of their measurement were adequate for their unambiguous interpretation. Measurements showed that the scattering geometry, conservation of energy, and the combinations of beam velocities and intersection angles for a given beam relative velocity were in accordance with the classical equations of motion and so verified the correct use of the instrumentation and the experimental approach. For all electron energies, the time coincidence detection of both electrons after energy analysis gives unique detection of free-free scattering and was used for all data reported here. It is essential to note the extensive development and characterization of the apparatus and the measurement techniques over many years [20-25] which has contributed to the successful performance of the present measurements.

Nevertheless, a unique feature of free electron-electron scattering of two electrons of any energy is that energy gain by either electron must have been transferred from the other electron in the absence of all other scattering process. Then the detection of a single electron at any energy and any angle, even without coincidence identification, identifies free-free electron scattering uniquely. Using unequal velocities of the beams, and then interchanging those velocities to obtain the same relative collision velocity provided an important experimental way of verifying the performance and symmetry of the measurements. The ability to change the relative velocity (i.e., the interaction energy) by changing the angle of intersection of the beams, while maintaining constant velocities (energies) of both beams, was a readily made check on measurement procedures and the scattering geometry, as seen in Fig. 2. Experimentally, variation of that angle rather than beam energies is preferable because the focal properties 


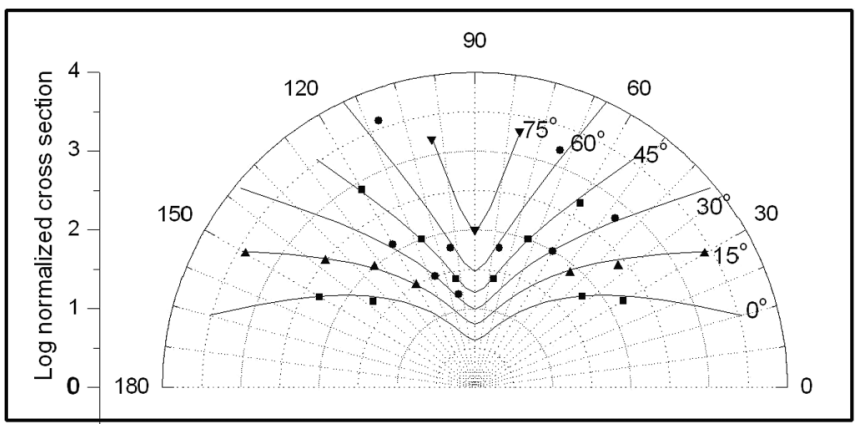

FIG. 4. The $\log _{10}$ of the differential cross sections $\left(\mathrm{cm}^{2} / \mathrm{sr}\right)$ is shown in angular (degrees) polar plots for the forward $\phi=0^{\circ}$ directions of the scattering angles from $0^{\circ}$ to $180^{\circ}$. The full lines are values calculated from the exact quantum equation (5) in the text for beam angles $\phi$ of $0^{\circ}, 15^{\circ}, 30^{\circ}, 45^{\circ}, 60^{\circ}$, and $75^{\circ}$ which are notedon each data set. The value of the $\log _{10}$ radial units is different for each curve and is for the same value of beam energy $E$. The data of each curve are normalized for nonoverlapping plots by the factor $\left(e^{2} / m V_{R}^{2}\right)^{2}$ of Eq. (5), i.e., $10^{-14} E^{-2} F$ (where $F=0.32,0.37,0.58$, $1.3,5.2,72$, respectively, for beam angles $\phi$ of $0^{\circ}, 15^{\circ}, 30^{\circ}, 45^{\circ}, 60^{\circ}$, $\left.75^{\circ}\right)$

of the electron beams remain constant and only the overlapping flux density changes. The changes of beam focal properties for large changes in beam energies were monitored and included in the beams' overlap calibrations of Eq. (4) which in turn contributed (via velocities and beam intersection angle) to the accuracy of the measurements. It is noted that measurement of the beams' overlap profile was straightforward for the charged electron beams compared with the inherent difficulties $[9,10]$ for noncharged beams.

\section{RESULTS}

The main results of this paper are shown in Figs. 4 and 5 for the differential cross sections $\left(\mathrm{cm}^{2} / \mathrm{sr}\right)$ as a function of scattering angle for selected beam interaction relative velocities. Numerous data at other beam intersection angles have been obtained and, rather than present many similar figures, a polar $\log _{10}$ graph format is used to present a systematic and compact visualization of the differential scattering cross sections. To obtain nonoverlapping plots in a single figure, the absolutely calibrated data are scaled (normalized) by $10^{-15} V_{R}^{4}$, that is by $E^{-2} \cos ^{2}(\phi)$ where $E$ is the common beam energy. The log of the differential cross sections (scaled radially) as functions of the scattering angles (polar circular axis) are shown for the forward $\phi=0^{\circ}$ direction in Fig. 4 and for the backward $\phi=180^{\circ}$ direction in Fig. 5. The $\log _{10}$ polar scale highlights the different effects of beam separation angles, i.e., relative velocities, on the observed cross sections.

The angular differential cross sections were measured as functions of the relative velocity of the interacting electron beams by varying (i) the beam intersection angles from $0^{\circ}, 15^{\circ}$, $30^{\circ}, 45^{\circ}, 60^{\circ}$, and $75^{\circ}$ and/or (ii) the energies of the incident beams; see Eq. (1). The reported data were obtained usually for simplicity of measurement from equal beam velocities (energies), as indicated in Fig. 2. For the given symmetric geometry of the initial intersecting equal velocity electrons

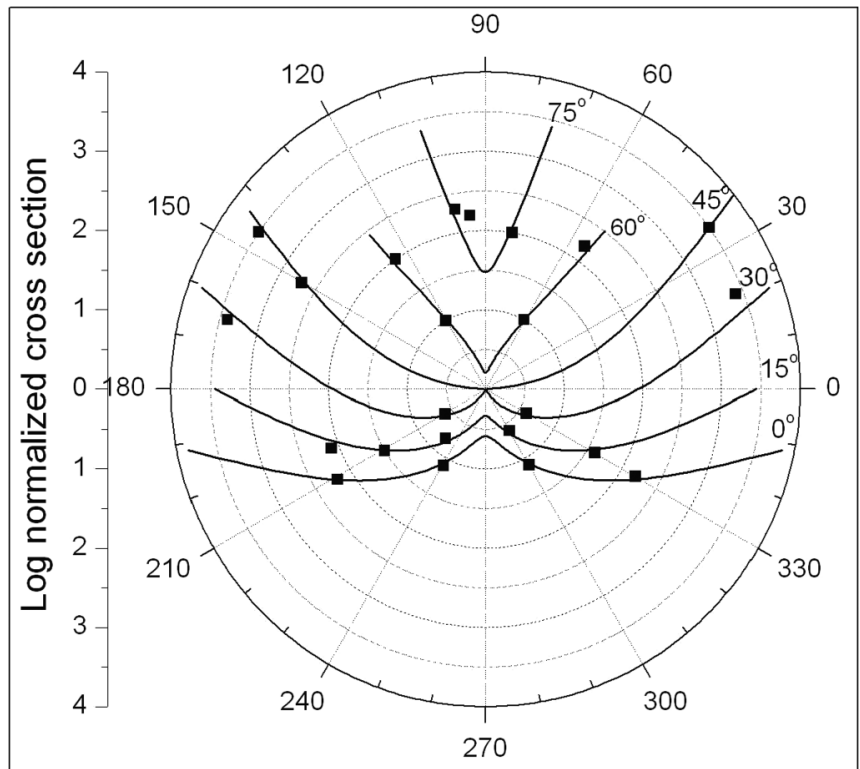

FIG. 5. The $\log _{10}$ of the differential cross sections is shown in angular (degrees) polar plots for the backward $\phi=180^{\circ}$ directions of the scattering angles from $0^{\circ}$ to $360^{\circ}$. Other information is the same as for Fig. 4.

and their reference frames, the symmetry of the observed scattering is well shown. The scattering angle of $90^{\circ}$, in the given laboratory coordinate frame and for equal energies of the incident electrons, was the direction of conservation of momentum of the incident electron beams. The difference between forward and backward scattering is apparent by comparing Figs. 4 and 5 for the same beam separation angles.

The energy spread in the laboratory system is proportional to the product of the beam velocities and the square of the relative beam divergence angle in the c.m. system. This feature is well known in merged (near zero intersection angle) beam measurements and will be reported separately [17] but here it is sufficient to indicate that they determined the range of beam velocities accepted by each electron energy analyzer. The related uncertainty is largest for the present studies when the measured cross section varies most rapidly with angle $\psi$, as implied from Fig. 2; however, it is less than the size of the plotted data points.

It is seen from Figs. 4 and 5 that the differential cross section follows the Rutherford $V_{R}^{4}$ velocity dependence of Eq. (5) within the measured uncertainties of $\pm 4 \%$. The additional time required for better precision was not pursued because the dependence was clear and consistent with Rutherford scattering. The $V_{R}^{4}$ factor is used effectively as a scaling parameter of the differential cross section and was used as such to guide the experimental choice of scattering parameters for acceptable scattered electron counting statistics.

The measured angular and energy differential cross sections in the laboratory $d \sigma(\theta, \phi) / d \Omega$ frame are compared with the theoretical differential cross sections calculated in the c.m. $d \sigma_{0}\left(\theta_{0}, \phi_{o}\right) / d \Omega_{0}$ frame using the exact Møller quantum formula averaged over the statistical electron spin distributions for unpolarized electrons [8] and neglecting spin effects. 
Then

$$
\begin{aligned}
d \sigma(\theta, \phi) / d \Omega= & {\left[d \sigma_{0}\left(\theta_{0}, \phi_{o}\right) / d \Omega_{0}\right] \Delta \Omega_{0} / \Delta \Omega } \\
= & \left(e^{2} / m V_{R}^{2}\right)^{2}\left(\operatorname{cosec}^{-4}\left(\theta_{0} / 2\right)+\sec ^{-4}\left(\theta_{0} / 2\right)\right. \\
& -\left[\operatorname{cosec}^{-2}\left(\theta_{0} / 2\right) \sec ^{-2}\left(\theta_{0} / 2\right)\right] \\
& \left.\times\left\{\cos \left[\left(e^{2} / \hbar V_{R}^{2}\right) \log \tan ^{2}\left(\theta_{0} / 2\right)\right]\right\}\right) \Delta \Omega_{0} / \Delta \Omega .
\end{aligned}
$$

The precision and reproducibility of the measurements were notably very good as reflected in Fig. 4 by the five data points which agree with the predicted Coulomb scattering predictions for combinations of beam angles of $15^{\circ}, 30^{\circ}, 45^{\circ}$, and $75^{\circ}$ for forward observation angles of $130^{\circ}, 60^{\circ}, 70^{\circ}, 111^{\circ}$, and $90^{\circ}$, respectively. Nevertheless, other data values (i) in Fig. 4 for forward scattering at the beam angle of $60^{\circ}$ for observations at $70^{\circ}$ and $110^{\circ}$ and (ii) in Fig. 5 for backward scattering at the beam angles of $60^{\circ}$ and $30^{\circ}$ for observations at $130^{\circ}$ and $20^{\circ}$, respectively, are more than up to a factor of 10 from the Coulomb values. Extensive testing did not determine the origin of those variations but we speculate that they arise most probably from beam angular deviations by surface charging. We report those values to indicate the unpredictability of experimental work and did not include them in our estimation of experimental uncertainties.

Consequently the main result of our work is that the present measurements, within their ranges of interacting velocities and scattering angles, have indicated the applicability of experiments to obtain agreement with the exact Møller formulation for pure Coulomb scattering, to describe free-free electron-electron scattering. Since the Møller formulation is exact it applies for all energies and angles and allows extrapolation of our data as required. It is noted also that the first Born approximation is obtained from Eq. (5) when the cosine term is unity and the classical description is obtained when the third term is zero.

Applications of the measured data are found in various plasmas in which electron-electron binary scattering is a way for the electrons to redistribute their energy to enable other energy-loss processes with different scattering probabilities to influence the rate of energy redistributions, for example, in dense high-temperature fully ionized plasmas, such as in the interiors of white dwarfs and neutron stars [2] which cannot be observed directly, or in quasifree atomic ionization processes with three or more outgoing electrons for which binary scattering may occur [26]. The data of Figs. 4 and 5, and Eq. (5), indicate that the largest cross sections are obtained as their relative velocity becomes smaller. Other different situations where binary scattering is influential occur in the initial state of three-body recombination and its inverse collisional ionization, and the final state of photo-double ionization as well as postcollision interactions [27]. For the three-body recombination process it has been claimed recently [28] that there are no viable theoretical approaches that explicitly calculate the wave functions for such states. Then, accurate cross section measurements are essential.

\section{CONCLUSIONS}

Direct measurement of the scattering of free electrons by free electrons was made using intersecting low-energy beams with scattered electron pairs identified uniquely by energy conservation and time coincidence detection. The method of changing the interaction velocity of the beams by changing the angle of intersection of the beams and/or changing the beam energies was shown to be applicable to the free electron-electron scattering. A significant conceptual feature of these binary collisions of equal mass charged particles, here electrons, is that, as the relative interaction velocity decreases, the center of mass of the outgoing particles moves away from the center of the laboratory coordinate frame and, in turn, results in a significant decrease of the ratio of the solid angles in the laboratory coordinate frame to those in the center-of-mass system. Consequently the observed scattering changes from forward and backward scattering to only forward scattering, i.e., in the direction of momentum transfer.

The exact spin-averaged Møller equation for pure Coulomb field scattering has been shown, within $4 \%$ uncertainty, to describe the measured energy and angle differential cross sections for free electron-electron scattering with dependencies of (relative velocity) ${ }^{-4}$ and $\cos ^{2}$ (beam intersection angle) scattering parameters for the incident electron interacting velocity range of $0.3 \times 10^{7}$ to $3.0 \times 10^{7} \mathrm{~m} / \mathrm{s}$.

The experimental approach warrants further studies of the scattering of equal mass particles, particularly at lower interacting velocities and with opposite charges, as well as merged electron-ion scattering phenomena, which will be reported separately.

\section{ACKNOWLEDGMENTS}

This research was supported by the University of Western Australia and by the Australian Research Council Centre of Excellence Program. O.A. is grateful to RFFI for the financial support of Grant No. 14-02-00386a. O.A. acknowledges Saint-Petersburg State University for a research grant, Grant No. 11.38.187.2014. O.T. and A.H. were supported by UWA undergraduate vacation scholarships. The mechanical scattering system was constructed and maintained by J. Budge, G. Light, and S. Key. The referees are thanked for clarification of the text.
[1] P. L. Anthony et al. (SLAC E158 Collaboration), Phys. Rev. Lett. 92, 181602 (2004).

[2] Y. D. Jung, Phys. Plasmas 8, 3842 (2001).

[3] D. K. Waterhouse and J. F. Williams, Phys. Rev. Lett. 79, 391 (1997).
[4] N. Andersen and K. Bartschat, Polarization, Alignment and Orientation in Atomic Collisions (Springer, New York, 2001).

[5] W. J. H. Leyland, G. H. John, R. T. Harley, M. M. Glazov, E. L. Ivchenko, D. A. Ritchie, I. Farrer, A. J. Shields, and M. Henini, Phys. Rev. B 75, 165309 (2007). 
[6] B. H. Bransden and C. J. Joachain, Physics of Atoms and Molecules, 2nd ed. (Prentice Hall, United Kingdom, 2003), p. 382.

[7] R. G. Parr and Y. Weitao, Density-Functional Theory of Atoms and Molecules (Oxford University Press, Oxford, 1994).

[8] N. F. Mott and H. S. W. Massey, The Theory of Atomic Collisions, 3rd ed. (Oxford University Press, Oxford, 1965), p. 817; C. Møller, Proc. R. Soc. A 12, 459 (1930).

[9] R. A. Phaneuf, C. C. Havener, G. H. Dunn, and A. Muller, Rep. Prog. Phys. 62, 1143 (1999).

[10] I. D. Williams, Rep. Prog. Phys. 62, 1431 (1999).

[11] K. T. Hecht, Quantum Mechanics (Springer, New York, 2000).

[12] J. F. Williams, J. Phys. B: At. Mol. Opt. Phys. 11, 2015 (1978).

[13] S. Samarin, O. M. Artamonov, V. N. Petrov, M. Kostylev, L. Pravica, A. Baraban, and J. F. Williams, Phys. Rev. B 84, 184433 (2011).

[14] S. N. Samarin, O. M. Artamonov, A. D. Sergeant, and J. F. Williams, Surf. Sci. 601, 4343 (2007).

[15] P. Hayes, M. A. Bennett, J. Flexman, and J. F. Williams, Phys. Rev. B 38, 13371 (1988).

[16] S. Samarin, O. M. Artamonov, A. D. Sergeant, A. A. Suvorova, and J. F. Williams, Solid State Commun. 129, 389 (2004).
[17] J. F. Williams (unpublished).

[18] J. H. Flexman, J. F. Williams, and P. Hayes, J. Phys. IV Colloq. 3C6, 79 (1993).

[19] E. L. Heck and J. F. Williams, J. Phys. B: At. Mol. Phys. 20, 2871 (1987).

[20] J. F. Williams and B. A. Willis, J. Phys. B: At. Mol. Phys. 7, L51 (1974).

[21] J. F. Williams and B. A. Willis, J. Phys. B: At. Mol. Phys. 8, 1670 (1975).

[22] J. F. Williams and B. A. Willis, J. Phys. B: At. Mol. Phys. 8, 1641 (1975).

[23] J. F. Williams and A. Crowe, J. Phys. B: At. Mol. Phys. 8, 2233 (1975).

[24] J. F. Williams, Phys. Rev. A 29, 2936(R) (1984).

[25] J. F. Williams, J. Phys. B: At. Mol. Phys. 21, 2107 (1988).

[26] A. Lahmam-Bennani, E. M. Staicu Casagrande, A. Naja, C. Dal Cappello, and P. Bolognesi, J. Phys. B: At. Mol. Opt. Phys. 43, 105201 (2010), and references therein.

[27] D. K. Waterhouse and J. F. Williams, J. Phys. B: At. Mol. Opt. Phys. 30, 2845 (1997).

[28] Y. K. Hahn, J. N. Gau, and E. Zerrad, Few Body Syst. 54, 1781 (2013). 